\title{
Travelling wave solutions of the generalized Zakharov-Kuznetsov equation via the extended generalized Riccati equation mapping method
}

\author{
Olusola T. Kolebaje ${ }^{1}{ }^{*}$, Pelumi Akinyemi ${ }^{1}$, Michael Obende ${ }^{2}$ \\ ${ }^{l}$ Department of Physics, Adeyemi College of Education, Ondo, Nigeria. \\ ${ }^{2}$ Department of Physics, Afe-Babalola University, Ado-Ekiti, Nigeria. \\ *Corresponding author E-mail:olusolakolebaje2008@gmail.com
}

\begin{abstract}
Exact hyperbolic, trigonometric and rational travelling wave solutions to the (2+1)-dimensional Generalized ZakharovKuznetsov (GZK) equation via the extended generalized Riccati equation mapping method are presented in this paper. The twenty one travelling wave solutions obtained were verified by putting them back into the GZK equation with the aid of Mathematica. This shows that the extended generalized Riccati equation mapping method is a powerful tool for finding exact solution to nonlinear partial differential equations in physics, mathematics and other applications.
\end{abstract}

Keywords: Extended generalized Riccati equation mapping method, Generalized Zakharov-Kuznetsov equation, nonlinear partial differential equations, Solitons, travelling wave solutions.

\section{Introduction}

In recent times, the calculation of exact travelling wave solution of nonlinear partial differential equations (NLPDE) play a very central role in soliton theory. These solutions provide much information about the physical models which the nonlinear partial differential equations describe. Nonlinear equations appear in many fields such as Quantum field theory, Optics, Plasma physics [1], Biology, Fluid mechanics [2], Solid-state physics [3], Chemical Kinetics [4], etc. Various powerful methods have been employed to construct exact solutions to nonlinear partial differential equations. These methods include the inverse scattering transform [5], the Backlund transform [6-7], the Darboux transform [8], the Hirota bilinear method [9], the tanh-function method [10-11], the sine-cosine method [12], the exp-function method [13], the generalized Riccati equation method [14], the Homogenous balance method [15], etc.

Recently a new method called the $\left(G^{\prime} / G\right)$ expansion method was introduced by [16] to construct exact solution to nonlinear partial differential equations. This method obtains travelling wave solution of NLPDE by using the second order differential equation given by $G^{\prime \prime}(\xi)+\lambda G^{\prime}(\xi)+\mu G(\xi)=0$ as an auxiliary equation.

Zayed and Khaled, [17] applied the $\left(G^{\prime} / G\right)$ expansion method to construct travelling wave solution of the combined $\mathrm{KdV}-\mathrm{mKdV}$ equation, the reaction-diffusion equation, the $\mathrm{KdV}$-Burgers equation and the generalized shallow water wave equation. Abdollahzadeh et al., [18] employed the $\left(G^{\prime} / G\right)$ expansion method for the exact travelling wave solution of the Benjamin-Bona-Mahony-Burgers equation. Higher order equations like the sixth order Ramani equation was also solved using the $\left(G^{\prime} / G\right)$ expansion method [19].

The extended generalized Riccati equation mapping method introduced by [20] is similar to the $\left(G^{\prime} / G\right)$ expansion method with the exception that the auxiliary equation used is the generalized Riccati equation given by $G^{\prime}(\xi)=p+$ $r G(\xi)+s G^{2}(\xi)$. The generalized Riccati equation has 27 different classes of solution which makes it ideal for constructing varieties of travelling wave solutions for other equations. Naher and Abdullah, [21] applied the extended generalized Riccati equation mapping method to construct new travelling wave solutions to the modified BenjaminBona-Mahony equation.

The aim of this paper is to apply the extended generalized Riccati equation mapping method to construct travelling wave solutions of the Generalized Zakharov-Kuznetsov (GZK) equation. 


\section{Description of the Extended Generalized Riccati Equation Mapping Method}

Here, we provide a brief explanation of the extended generalized Riccati equation mapping method for finding travelling wave solutions of nonlinear partial differential equations. Consider a nonlinear equation of two independent variables $x$ and $t$ of the form:

$P\left(u, u_{t}, u_{x}, u_{t t}, u_{x x}, u_{x t}, \ldots\right)=0$

$P$ is a polynomial in $u(x, t)$ and its derivatives with respect to $x$ and $t$. To begin, we transform equation (1) into a nonlinear ordinary differential equation by introducing the variable $\xi$ given by:

$u(x, t)=u(\xi) \quad \xi=\mu x+c t$

Where $\mu$ and $c$ are arbitrary constant and equation (2) reduces to a nonlinear ordinary differential equation of the form

$Q\left(u, u^{\prime}, u^{\prime \prime}, u^{\prime \prime \prime} \ldots\right)=0$

The method assumes that the solution to equation (1) can be expressed as a polynomial in $\left(G^{\prime} / G\right)$

$u(\xi)=\sum_{i=0}^{m} \alpha_{i}\left(\frac{G^{\prime}}{G}\right)^{i} \quad \alpha_{m} \neq 0$

Where $\alpha_{0}, \alpha_{1}, \ldots, \alpha_{m}$ are constants to be determined and $G(\xi)$ satisfies the generalized Riccati equation of the form

$G^{\prime}(\xi)=p+r G(\xi)+s G^{2}(\xi) \quad s \neq 0$

Where $p, r$ and $s$ are arbitrary constants. Equation (3) is integrated as long as all the terms contain derivatives, where integration constants are considered to be zero. To determine $m$, we consider the homogenous balance between the highest order derivative and the highest order nonlinear term(s).

Substitute equation (4) with the determined value of $m$ into equation (3), and collect all terms with the same order of $G^{-m}(\xi)$ and $G^{m}(\xi)$ for $m=0,1,2,3, \ldots$ together. If the coefficients of $G^{m}$ vanish separately, we have a set of algebraic equations in $\alpha_{0}, \alpha_{1}, \ldots, \alpha_{m}, \mu, c, p, r$ and $s$ that is solved with the aid of Mathematica.

Finally, substituting $\alpha_{0}, \alpha_{1}, \ldots, \alpha_{m}, c$ and the general solution to equation (5) into equation (4) yield the travelling wave solution of equation (1). Twenty seven different solutions of the generalized Riccati equation under four different families are presented below [21]:

Family 1: When $\phi=\sqrt{r^{2}-4 s p}, \phi^{2}>0$ and $r s \neq 0$ or $s p \neq 0$, the hyperbolic solutions to equation (5) are:

$$
\begin{aligned}
& G_{1}=-\frac{1}{2 s}\left[r+\phi \tanh \left(\frac{\phi}{2} \xi\right)\right] \\
& G_{2}=-\frac{1}{2 s}\left[r+\phi \operatorname{coth}\left(\frac{\phi}{2} \xi\right)\right] \\
& G_{3}=-\frac{1}{2 s}[r+\phi[\tanh (\phi \xi) \pm i \operatorname{sech}(\phi \xi)]] \\
& G_{4}=-\frac{1}{2 s}[r+\phi[\operatorname{coth}(\phi \xi) \pm \operatorname{cosech}(\phi \xi)]] \\
& G_{5}=-\frac{1}{4 s}\left[2 r+\phi\left[\tanh \left(\frac{\phi}{4} \xi\right)+\operatorname{coth}\left(\frac{\phi}{4} \xi\right)\right]\right] \\
& G_{6}=\frac{1}{2 s}\left[-r+\frac{\phi \sqrt{Q^{2}+R^{2}}-Q \phi \cosh (\phi \xi)}{Q \sinh (\phi \xi)+R}\right] \\
& G_{7}=\frac{1}{2 s}\left[-r-\frac{\phi \sqrt{R^{2}-Q^{2}}+Q \phi \sinh (\phi \xi)}{Q \cosh (\phi \xi)+R}\right]
\end{aligned}
$$


$Q$ and $R$ are two non-zero real constants that satisfies $R^{2}-Q^{2}>0$.

$$
\begin{aligned}
G_{8} & =\frac{2 p \cosh \left(\frac{\phi}{2} \xi\right)}{\phi \sinh \left(\frac{\phi}{2} \xi\right)-r \cosh \left(\frac{\phi}{2} \xi\right)} \\
G_{9} & =\frac{-2 p \sinh \left(\frac{\phi}{2} \xi\right)}{-\phi \cosh \left(\frac{\phi}{2} \xi\right)+r \sinh \left(\frac{\phi}{2} \xi\right)} \\
G_{10} & =\frac{2 p \cosh (\phi \xi)}{\phi \sinh (\phi \xi)-r \cosh (\phi \xi) \pm i \phi} \\
G_{11} & =\frac{2 p \sinh (\phi \xi)}{\phi \cosh (\phi \xi)-r \sinh (\phi \xi) \pm \phi} \\
G_{12} & =\frac{4 p \sinh \left(\frac{\phi}{4} \xi\right) \cosh \left(\frac{\phi}{4} \xi\right)}{2 \phi \cosh ^{2}\left(\frac{\phi}{4} \xi\right)-2 r \sinh \left(\frac{\phi}{4} \xi\right) \cosh \left(\frac{\phi}{4} \xi\right)-\phi}
\end{aligned}
$$

Family 2: When $\eta=\sqrt{4 s p-r^{2}}, \eta^{2}>0$ and $r s \neq 0$ or $s p \neq 0$, the trigonometric solutions to equation (5) are:

$$
\begin{aligned}
& G_{13}=\frac{1}{2 s}\left[-r+\eta \tan \left(\frac{\eta}{2} \xi\right)\right] \\
& G_{14}=-\frac{1}{2 s}\left[r+\eta \cot \left(\frac{\eta}{2} \xi\right)\right] \\
& G_{15}=\frac{1}{2 s}[-r+\eta[\tan (\eta \xi) \pm \sec (\eta \xi)]] \\
& G_{16}=-\frac{1}{2 s}[r+\eta[\cot (\eta \xi) \pm \operatorname{cosec}(\eta \xi)]] \\
& G_{17}=\frac{1}{4 s}\left[-2 r+\eta\left[\tan \left(\frac{\eta}{4} \xi\right)-\cot \left(\frac{\eta}{4} \xi\right)\right]\right] \\
& G_{18}=\frac{1}{2 s}\left[-r+\frac{ \pm \eta \sqrt{Q^{2}-R^{2}}-Q \eta \cos (\eta \xi)}{Q \sin (\eta \xi)+R}\right] \\
& G_{19}=\frac{1}{2 s}\left[-r-\frac{ \pm \eta \sqrt{Q^{2}-R^{2}}+Q \eta \cos (\eta \xi)}{Q \sin (\eta \xi)+R}\right]
\end{aligned}
$$

$Q$ and $R$ are two non-zero real constants that satisfies $Q^{2}-R^{2}>0$.

$$
\begin{aligned}
& G_{20}=\frac{-2 p \cos \left(\frac{\eta}{2} \xi\right)}{\eta \sin \left(\frac{\eta}{2} \xi\right)+r \cos \left(\frac{\eta}{2} \xi\right)} \\
& G_{21}=\frac{2 p \sin \left(\frac{\eta}{2} \xi\right)}{\eta \cos \left(\frac{\eta}{2} \xi\right)-r \sin \left(\frac{\eta}{2} \xi\right)} \\
& G_{22}=\frac{-2 p \cos (\eta \xi)}{\eta \sin (\eta \xi)+r \cos (\eta \xi) \pm \eta} \\
& G_{23}=\frac{2 p \sin (\eta \xi)}{\eta \cos (\eta \xi)-r \sin (\eta \xi) \pm \eta} \\
& G_{24}=\frac{4 p \sin \left(\frac{\eta}{4} \xi\right) \cos \left(\frac{\eta}{4} \xi\right)}{2 \eta \cos ^{2}\left(\frac{\eta}{4} \xi\right)-2 r \sin \left(\frac{\eta}{4} \xi\right) \cosh \left(\frac{\eta}{4} \xi\right)-\eta}
\end{aligned}
$$

Family 3: When $p=0, r s \neq 0$ and $g_{1}$ is an arbitrary constant, then we have more solutions to equation (5): 
$G_{25}=\frac{-r g_{1}}{s\left[g_{1}+\cosh (r \xi)-\sinh (r \xi)\right]}$

$G_{26}=\frac{-r[\cosh (r \xi)+\sinh (r \xi)]}{s\left[g_{1}+\cosh (r \xi)+\sinh (r \xi)\right]}$

Family 4: When $p=r=0, s \neq 0$ and $d_{1}$ is an arbitrary constant, then the rational solutions to equation (5) are:

$G_{27}=\frac{-1}{s \xi+d_{1}}$

\section{Application}

In this section, we apply the extended generalized Riccati equation mapping method to construct travelling wave solution of the (2+1)-dimensional Generalized Zakharov-Kuznetsov (GZK) equation given by [22-23],

$u_{t}+a u u_{x}+b u^{2} u_{x}+c u_{x x x}+d u_{x y y}=0$

Where $a, b, c$ and $d$ are constants and $b \neq 0$. This equation describes the nonlinear development of ion-acoustic waves in magnetized plasma under the restrictions of small wave amplitude, weak dispersion, and strong magnetic fields [24].

We make the transformation $(x, y, t)=u(\xi), \xi=\mu x+\beta y+\omega t$.

Equation (6) becomes

$\omega u^{\prime}+a \mu u u^{\prime}+b \mu u^{2} u^{\prime}+\left(c \mu^{3}+d \beta^{2} \mu\right) u^{\prime \prime \prime}=0$

Integrating equation (7) once with respect to $\xi$ yields

$\omega u+\frac{a \mu}{2} u^{2}+\frac{b \mu}{3} u^{3}+\left(c \mu^{3}+d \beta^{2} \mu\right) u^{\prime \prime}+k=0$

Where $k$ is a constant of integration to be determined later. To get $m$, we balance the highest order derivative $u^{\prime \prime}$ and the highest order nonlinear term $u^{3}$.

$3 m=m+2 \Rightarrow m=1$

Then equation (4) becomes

$u(\xi)=\alpha_{1}\left(\frac{G^{\prime}}{G}\right)+\alpha_{0} \quad$ where $\alpha_{1} \neq 0$

Where $\alpha_{0}$ and $\alpha_{1}$ are constants to be determined later. Substituting equation (5) in equation (9)

$u(\xi)=\alpha_{1}\left(p G^{-1}+r+s G\right)+\alpha_{0}$

Substituting equation (10) and its derivatives into equation (8) and collecting the coefficients of $G^{ \pm m}(\xi)$ together yields a simultaneous set of nonlinear algebraic equations in $\alpha_{0}, \alpha_{1}, r, s, p, \omega$ and $k$. These equations are not displayed to save space due to their long nature.

Solving this algebraic system of equation with the aid of Mathematica yields the solution

$$
\begin{aligned}
& \alpha_{0}=-\frac{a}{2 b} \pm r \sqrt{\frac{-3 d \beta^{2}-3 c \mu^{2}}{2 b}} \quad \alpha_{1}=\mp 2 \sqrt{\frac{-3 d \beta^{2}-3 c \mu^{2}}{2 b}} \\
& \omega=\frac{1}{4}\left[\frac{a^{2} \mu}{b}+2 d r^{2} \beta^{2} \mu+16 d p s \beta^{2} \mu+2 c r^{2} \mu^{3}+16 c p s \mu^{3}\right]
\end{aligned}
$$


$k=\frac{\mu}{24 b^{2}}\left[a^{3}-48 \sqrt{6} b^{3 / 2} \operatorname{prs}\left(-d \beta^{2}-c \mu^{2}\right)^{3 / 2}+6 a b\left(r^{2}+8 s p\right)\left(d \beta^{2}+c \mu^{2}\right)\right]$

Substituting the solution to the nonlinear algebraic equation and the general solution to the generalized Riccati equation (5) into equation (9), we obtain different travelling wave solutions of the GZK equation.

Family 1: The hyperbolic solutions to equation (6), when $\phi=\sqrt{r^{2}-4 s p}, \phi^{2}>0$ and $r s \neq 0$ or $s p \neq 0$ are:

$$
\begin{aligned}
& \alpha_{0}=-\frac{a}{2 b} \pm r \sqrt{\frac{-3 d \beta^{2}-3 c \mu^{2}}{2 b}} \quad \alpha_{1}=\mp 2 \sqrt{\frac{-3 d \beta^{2}-3 c \mu^{2}}{2 b}} \\
& \xi=\mu x+\beta y+\frac{1}{4}\left[\frac{a^{2} \mu}{b}+2 d r^{2} \beta^{2} \mu+16 d p s \beta^{2} \mu+2 c r^{2} \mu^{3}+16 c p s \mu^{3}\right] t \\
& u_{1}(\xi)=\alpha_{1}\left(\frac{\phi^{2} \operatorname{sech}^{2}\left(\frac{\phi}{2} \xi\right)}{2\left[r+\phi \tanh \left(\frac{\phi}{2} \xi\right)\right]}\right)+\alpha_{0} \\
& u_{2}(\xi)=\alpha_{1}\left(\frac{-\phi^{2} \operatorname{cosech}^{2}\left(\frac{\phi}{2} \xi\right)}{2\left[r+\phi \operatorname{coth}\left(\frac{\phi}{2} \xi\right)\right]}\right)+\alpha_{0} \\
& u_{3}(\xi)=\alpha_{1}\left(\frac{ \pm i \phi^{2}}{\phi \cosh (\phi \xi)+r[ \pm i+\sinh (\phi \xi)]}\right)+\alpha_{0} \\
& u_{4}(\xi)=\alpha_{1}\left(\frac{\phi^{2}}{r \mp r \cosh (\phi \mu) \mp \phi \sinh (\phi \mu)}\right)+\alpha_{0} \\
& u_{5}(\xi)=\alpha_{1}\left(\frac{-\phi^{2} \operatorname{cosech}^{2}\left(\frac{\phi}{2} \xi\right)}{2 r+\phi\left[\operatorname{coth}\left(\frac{\phi}{4} \xi\right)+\tanh \left(\frac{\phi}{4} \xi\right)\right]}\right)+\alpha_{0} \\
& u_{6}(\xi)=\alpha_{1}\left(\frac{Q\left[-Q \phi^{2}+\phi^{2} \sqrt{Q^{2}+R^{2}} \cosh (\phi \xi)+R \phi^{2} \sinh (\phi \xi)\right]}{[R+Q \sinh (\phi \xi)]\left[r R-\phi \sqrt{Q^{2}+R^{2}}+Q \phi \cosh (\phi \xi)+Q r \sinh (\phi \xi)\right]}\right)+\alpha_{0} \\
& u_{7}(\xi)=\alpha_{1}\left(\frac{Q\left[Q \phi^{2}-\phi^{2} \sqrt{R^{2}-Q^{2}} \sinh (\phi \xi)+R \phi^{2} \cosh (\phi \xi)\right]}{[R+Q \cosh (\phi \xi)]\left[r R+\phi \sqrt{R^{2}-Q^{2}}+Q \phi \sinh (\phi \xi)+Q r \cosh (\phi \xi)\right]}\right)+\alpha_{0} \\
& u_{8}(\xi)=\alpha_{1}\left(\frac{\phi^{2}}{r+r \cosh (\phi \xi)-\phi \sinh (\phi \xi)}\right)+\alpha_{0} \\
& u_{9}(\xi)=\alpha_{1}\left(\frac{\phi^{2}}{r-r \cosh (\phi \xi)+\phi \sinh (\phi \xi)}\right)+\alpha_{0} \\
& u_{10}(\xi)=\alpha_{1}\left(\frac{\phi^{2}[\operatorname{sech}(\phi \xi) \mp i \tanh (\phi \xi)]}{r \cosh (\phi \xi)-\phi[ \pm i+\sinh (\phi \xi)]}\right)+\alpha_{0} \\
& u_{11}(\xi)=\alpha_{1}\left(\frac{\phi^{2}}{r \mp r \cosh (\phi \xi) \pm \phi \sinh (\phi \xi)}\right)+\alpha_{0} \\
& u_{12}(\xi)=\alpha_{1}\left(\frac{\phi^{2}}{r-r \cosh (\phi \xi)+\phi \sinh (\phi \xi)}\right)+\alpha_{0}
\end{aligned}
$$

Family 2: The trigonometric solutions to equation (6), when $\eta=\sqrt{4 s p-r^{2}}, \eta^{2}>0$ and $r s \neq 0$ or $s p \neq 0$ are:

$$
\begin{aligned}
& \alpha_{0}=-\frac{a}{2 b} \pm r \sqrt{\frac{-3 d \beta^{2}-3 c \mu^{2}}{2 b}} \quad \alpha_{1}=\mp 2 \sqrt{\frac{-3 d \beta^{2}-3 c \mu^{2}}{2 b}} \\
& \xi=\mu x+\beta y+\frac{1}{4}\left[\frac{a^{2} \mu}{b}+2 d r^{2} \beta^{2} \mu+16 d p s \beta^{2} \mu+2 c r^{2} \mu^{3}+16 c p s \mu^{3}\right] t
\end{aligned}
$$




$$
\begin{aligned}
& u_{13}(\xi)=\alpha_{1}\left(\frac{-\eta^{2} \sec ^{2}\left(\frac{\eta}{2} \xi\right)}{2\left[r-\eta \tan \left(\frac{\eta}{2} \xi\right)\right]}\right)+\alpha_{0} \\
& u_{14}(\xi)=\alpha_{1}\left(\frac{-\eta^{2} \operatorname{cosec}^{2}\left(\frac{\eta}{2} \xi\right)}{2\left[r+\eta \cot \left(\frac{\eta}{2} \xi\right)\right]}\right)+\alpha_{0} \\
& u_{15}(\xi)=\alpha_{1}\left(\frac{-\eta^{2}}{r \mp \eta \cos (\eta \xi) \mp r \sin (\eta \xi)}\right)+\alpha_{0} \\
& u_{16}(\xi)=\alpha_{1}\left(\frac{-\eta^{2}}{r \pm \eta \sin (\eta \xi) \mp r \cos (\eta \xi)}\right)+\alpha_{0} \\
& u_{17}(\xi)=\alpha_{1}\left(\frac{-\eta^{2} \operatorname{cosec}^{2}\left(\frac{\eta}{2} \xi\right)}{2 r+\eta\left[\cot \left(\frac{\eta}{4} \xi\right)-\tan \left(\frac{\eta}{4} \xi\right)\right]}\right)+\alpha_{0} \\
& u_{18}(\xi)=\alpha_{1}\left(\frac{Q\left[-Q \eta^{2} \pm \eta^{2} \sqrt{Q^{2}-R^{2}} \cos (\eta \xi)-R \eta^{2} \sin (\eta \xi)\right]}{[R+Q \sin (\eta \xi)]\left[r R \mp \eta \sqrt{Q^{2}-R^{2}}+Q \eta \cos (\eta \xi)+Q r \sin (\eta \xi)\right]}\right)+\alpha_{0} \\
& u_{19}(\xi)=\alpha_{1}\left(\frac{Q\left[-Q \eta^{2} \mp \eta^{2} \sqrt{Q^{2}-R^{2}} \cos (\eta \xi)-R \eta^{2} \sin (\eta \xi)\right]}{[R+Q \sin (\eta \xi)]\left[r R \pm \eta \sqrt{Q^{2}-R^{2}}+Q \eta \cos (\eta \xi)+Q r \sin (\eta \xi)\right]}\right)+\alpha_{0} \\
& u_{20}(\xi)=\alpha_{1}\left(\frac{-\eta^{2}}{r+r \cos (\eta \xi)+\eta \sin (\eta \xi)}\right)+\alpha_{0} \\
& u_{21}(\xi)=\alpha_{1}\left(\frac{-\eta^{2}}{r-r \cos (\eta \xi)-\eta \sin (\eta \xi)}\right)+\alpha_{0} \\
& u_{22}(\xi)=\alpha_{1}\left(\frac{-\eta^{2}[\sec (\eta \xi) \pm \tan (\eta \xi)]}{r \cos (\eta \xi)+\eta[ \pm 1+\sin (\eta \xi)]}\right)+\alpha_{0} \\
& u_{23}(\xi)=\alpha_{1}\left(\frac{-\eta^{2}}{r \mp r \cos (\eta \xi) \mp \eta \sin (\eta \xi)}\right)+\alpha_{0} \\
& u_{24}(\xi)=\alpha_{1}\left(\frac{-\eta^{2}}{r-r \cos (\eta \xi)-\eta \sin (\eta \xi)}\right)+\alpha_{0}
\end{aligned}
$$

Family 3: More solutions to equation (6), when $p=0, r s \neq 0$ and $g_{1}$ is an arbitrary constant are:

$$
\begin{aligned}
& \alpha_{0}=-\frac{a}{2 b} \pm r \sqrt{\frac{-3 d \beta^{2}-3 c \mu^{2}}{2 b}} \quad \alpha_{1}=\mp 2 \sqrt{\frac{-3 d \beta^{2}-3 c \mu^{2}}{2 b}} \\
& \xi=\mu x+\beta y+\frac{1}{4}\left[\frac{a^{2} \mu}{b}+2 d r^{2} \beta^{2} \mu+2 c r^{2} \mu^{3}\right] t \\
& u_{25}(\xi)=\alpha_{1}\left(\frac{r}{1+e^{r \xi} g_{1}}\right)+\alpha_{0} \\
& u_{26}(\xi)=\alpha_{1}\left(\frac{r g_{1}}{e^{r \xi}+g_{1}}\right)+\alpha_{0}
\end{aligned}
$$

Family 4: Rational solutions to equation (6), when $p=r=0, s \neq 0$ and $d_{1}$ is an arbitrary constant are:

$$
\begin{aligned}
& \alpha_{0}=-\frac{a}{2 b} \pm r \sqrt{\frac{-3 d \beta^{2}-3 c \mu^{2}}{2 b}} \quad \alpha_{1}=\mp 2 \sqrt{\frac{-3 d \beta^{2}-3 c \mu^{2}}{2 b}} \\
& \xi=\mu x+\beta y+\frac{a^{2} \mu}{4 b} t \\
& u_{27}(\xi)=\alpha_{1}\left(\frac{-s}{s \xi+d_{1}}\right)+\alpha_{0}
\end{aligned}
$$




\section{Results and discussion.}

Remark 4.1: The travelling wave solutions of the (2+1)-dimensional Generalized Zakharov-Kuznetsov (GZK) equation obtained using the extended generalized Riccati equation mapping method for the hyperbolic, trigonometric and rational function types are presented in $u_{1}-u_{27}$.

Remark 4.2: The solutions $u_{8}, u_{9}, u_{11}$ and $u_{12}$ are identical and can all be described by $u_{11}$ alone. Also solutions $u_{20}, u_{21}, u_{23}$ and $u_{24}$ are identical and can all be described by solution $u_{23}$. Hence the extended generalized Riccati equation mapping method produces 21 different classes of travelling wave solution to the GZK equation. This compared to 8 classes of solutions to the GZK equation reported by [24] using the improved $\left(G^{\prime} / G\right)$ expansion method shows the superiority of the extended generalized Riccati equation mapping method.

Remark 4.3: All the obtained travelling wave solutions to the (2+1)-dimensional (GZK) equation $u_{1}-u_{7}, u_{11}, u_{13}-$ $u_{19}, u_{23}$ and $u_{25}-u_{27}$ were checked by putting them back into equation (6) with the aid of Mathematica.

\section{Conclusion}

Twenty one different hyperbolic, trigonometric and rational function travelling wave solutions to the (2+1)-dimensional Generalized Zakharov-Kuznetsov (GZK) equation has been obtained using the extended generalized Riccati equation mapping method. The results have been verified by putting them back into the GZK equation with the aid of Mathematica. Conclusively, the extended generalized Riccati equation mapping method is a powerful tool for finding exact solution to nonlinear partial differential equations in physics, mathematics and other fields.

\section{References}

[1] A. Hasegawa. Plasma Instabilities and Nonlinear Effects. Berlin: Springer-Verlag, 1975.

[2] G. Whitham. Linear and Nonlinear Waves. New York: Wiley, 1974.

[3] G. Eilenberger. Solitons. Berlin: Springer-Verlag, 1983.

[4] P. Gray and S. Scott. Chemical Oscillations and Instabilities. Oxford: Clarendon, 1990.

[5] M.J. Ablowitz, H. Segur. Solitons and the inverse scattering transform. Philadelphia: SIAM, 1981.

[6] M.R. Miura. Backlund Transformation. Berlin: Springer-Verlag, 1978.

[7] C. Rogers, W.F. Shadwick. Backlund Transformations. New York: Academic Press, 1982.

[8] V. B. Matveev, M. A. Salle. Darboux transformations and solitons. Berlin: Springer, 1991.

[9] R. Hirota, Exact envelope soliton solutions of a nonlinear wave equation. J. Math. Phys. 1973; 14: 805-810.

[10] E.G. Fan, Extended tanh-function method and its applications to nonlinear equations. Phys. Lett. A 2000; 277: 212-218.

[11] Malfliet, W., The tanh method: a tool for solving certain classes of non-linear PDEs. Mathematical Methods in the Applied Sci. 2005; 28 (17): 2031-2035.

[12] C.T. Yan, A simple transformation for nonlinear waves. Phys. Lett. A 1996; 224: 77-84.

[13] J.H. He and X.H. Wu, Construction of solitary solution and compacton-like solution by variational iteration method. Chaos, Solitons and Fractals 2006; 29 (1): 108-113.

[14] Z.Y. Yan and H.Q. Zhang, New explicit solitary wave solutions and periodic wave solutions for Whitham-Broer-Kaup equation in shallow water. Phys. Lett. A 2001; 285: 355-362.

[15] E.G. Fan, Two new applications of the homogeneous balance method. Phys. Lett. A 2000; 265: 353-357.

[16] Wang, M.L., Li, X., and Zhang, J., The (G'/G)-expansion method and traveling wave solutions of nonlinear evolution equations in mathematical physics. Phys. Lett. A 2008; 372: 417-423.

[17] Zayed E.M.E. and Khaled A.G., The (G'/G) expansion method for finding travelling wave solutions of nonlinear partial differential equations in mathematical physics. Journal of Mathematical Physics 2009; 50 (1): 1-12.

[18] M. Abdollahzadeh, M. Hosseini, M. Ghanbarpour, and S. Kashani, Exact travelling solution for Benjamin-Bona-Mahony-Burgers equation by $\left(\mathrm{G}^{\prime} / \mathrm{G}\right)$ expansion method. International Journal of Applied Mathematics and Computation 2011; 3 (1): 70-76.

[19] Ibrahim E.I., (G'/G) expansion method for travelling wave solution of the sixth-order Ramani equation. Cankaya University Journal of Science and Engineering 2010; 7 (1): 51-57.

[20] S. Zhu, The Generalizing Riccati Equation Mapping Method In Non-Linear Evolution Equation: Application To (2+1)-Dimensional BoitiLeon-Pempinelle Equation. Chaos, Solitons and Fractals 2008; 37: 1335-1342.

[21] H. Naher and F.A. Abdullah, The Modified Benjamin-Bona-Mahony Equation via the Extended Generalized Riccati Equation Mapping Method. Applied Mathematical Sciences 2012; 6 (111): 5495 - 5512.

[22] V.E. Zakharov and E.M. Kuznetsov, On three-dimensional solitons. Soviet Phys. JETP 1974; 39: $285-293$.

[23] M.H.M. Moussa, Similarity solutions to non-linear partial differential equationof physical phenomena represented by the Zakharov-Kuznetsov equation. Int. J. Eng. Sci. 2001; 39: 1565-1575.

[24] Yanhong Q. and Baodan T., Generalized G'/G-Expansion Method and its Applications. International Mathematical Forum 2011 ; 6 (3): 147 157. 